\title{
Clinicopathological Significance of S100A10 Expression in Lung Adenocarcinomas
}

\author{
Ken Katono ${ }^{1}$, Yuichi Sato ${ }^{2 *}$, Shi-Xu Jiang ${ }^{3}$, Makoto Kobayashi ${ }^{2}$, Keita Saito ${ }^{2}$, \\ Ryo Nagashio ${ }^{2}$, Shinichiro Ryuge ${ }^{1}$, Yukitoshi Satoh $^{4}$, Makoto Saegusa ${ }^{3}$, Noriyuki \\ Masuda $^{1}$
}

\begin{abstract}
Background: S100A10, of the S100 protein family, is reported to be involved in cancer cell invasion and metastasis. The aims of the present study were to immunohistochemically examine S100A10 expression in surgically resected lung adenocarcinomas, and evaluate any relationships with clinicopathological parameters and prognosis of patients. Materials and Methods: S100A10 expression was immunohistochemically studied in 202 consecutive resected lung adenocarcinomas, and its associations with clinicopathological parameters were evaluated. Kaplan-Meier survival analysis and Cox proportional hazards models were used to estimate the effect of S100A10 expression on survival. Results: S100A10 expression was detected in 65 of the $202(32.2 \%)$ lung adenocarcinomas, being significantly correlated with poorer differentiation $(P=0.015)$, a higher pathological TNM stage (stages II and III) (P=0.004), more frequent and severe intratumoral vascular invasion (P=0.001), and a poorer prognosis $(\mathrm{P}=\mathbf{0 . 0 3 0})$. However, $\mathrm{S100A10}$ expression was not an independent predictor of survival after

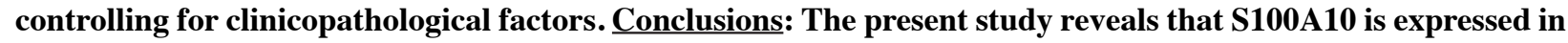
a subset of lung adenocarcinomas, and this is related to some clinicopathological parameters, although further studies are required to confirm the correlation between $\mathrm{S100A10}$ expression and prognosis of lung adenocarcinoma patients.
\end{abstract}

Keywords: S100A10 - plasminogen receptor - lung adenocarcinoma - prognosis

Asian Pac J Cancer Prev, 17 (1), 289-294

\section{Introduction}

Non-small cell lung cancer (NSCLC) is the leading cause of cancer-related mortality worldwide, and lung adenocarcinoma accounts for about a half of NSCLCs (Parkin, 2001; Alberg et al., 2013). Despite advances in surgical techniques, the 5-year survival rates of patients with surgically resectable NSCLC has only slightly improved over the last few decades (Yoshino et al., 2012). Furthermore, molecular targeted therapies such as epidermal growth factor receptor (EGFR) -tyrosine kinase inhibitors demonstrated high-level efficacy in patients with lung adenocarcinoma harboring EGFR mutations, although acquired resistance develops an average of 12 months after the initiation of therapy (Kobayashi et al., 2005). Thus, further understanding of the tumorigenesis and tumor biology of lung adenocarcinoma is crucial in order to develop novel therapeutic strategies to improve patients' prognoses.

The S100 protein family comprises calcium-binding protein of the EF-hand type which consists of at least 25 distinct members, and plays roles including intra- and extracellular functions involved in the cell cycle, growth, migration, and protein phosphorylation (Donato, 2001). Recently, the S100 protein family has been thought to play key roles during different steps of the tumorigenic processes and tumor progression (Chen et al., 2014). Moreover, expression of the $\mathrm{S} 100$ protein family is detected in many human cancers and has been related to a poorer prognosis. In lung cancer, S100A2, S100A4, and S100A9 expressions were related to a poorer prognosis and suggested to be prognostic markers (Wang et al., 2005; Tsuna et al., 2009; Kawai et al., 2011).

$\mathrm{S} 100 \mathrm{~A} 10$ is also a member of the $\mathrm{S} 100$ protein family, and it forms a heterotetramer with annexinA2, which functions as a plasminogen receptor on the cell surface (Godier and Hunt, 2013). Plasminogen converts plasmin through binding to its receptors including the S100A10annexinA2 heterotetramer, and plasmin catalyzes the degradiation of proteins of the basement membrane and extracellular matrix (ECM) (Hedhli et al., 2012; Liu et al., 2015). Increasing plasmin production has been shown to enhance the capability of cancer cells to degrade proteins of the basement membrane and ECM, which facilitated the infiltration of tumor cells into surrounding tissues (Godier and Hunt, 2013; Madureira 
et al., 2012). The overexpression of S100A10 is observed in renal cell carcinoma and anaplastic thyroid carcinoma (Domoto et al., 2007; Ito et al., 2007). Furthermore, the overexpression of S100A10 is related to poorer prognoses in gallbladder and colorectal cancer patients (Tan et al., 2011; Shang et al., 2013). However, to our knowledge, no report is available concerning the significance of S100A10 expression in the clinicopathological features and prognoses in a large series of lung adenocarcinoma patients. The present study examined S100A10 expression in resected lung adenocarcinomas and analyzed the correlation with clinicopathological parameters and its prognostic significance.

\section{Materials and Methods}

\section{Patients and tissue specimens}

A total of 202 consecutive lung adenocarcinoma patients who underwent complete resection from January 2002 to December 2005 at Kitasato University Hospital were included in this retrospective cohort study. Those who received preoperative chemotherapy and/or radiotherapy were excluded. Ten percent formalin-fixed and paraffin-embedded tissues were processed into $3-\mu \mathrm{m}-$ thick sections and stained with hematoxylin and eosin. The histological diagnosis was based on the criteria of the World Health Organization/International Association for the Study of Lung Cancer (Travis et al., 1999). Each case was reassessed according to the $7^{\text {th }}$ edition of the TNM classification (Goldstraw et al., 2007). The clinical and pathologic parameters retrospectively reviewed included the age at surgical resection, sex, smoking habit, tumor differentiation, nodal status, intratumoral vascular invasion, intratumoral lymphatic invasion, pleural invasion, pathological TNM (p-TNM) and stage, receiving adjuvant chemotherapy, viability status, and survival time after surgery. The viability status was determined based on whether or not lung cancer-related death occurred, and the survival time was defined as the duration from the date of surgery to that of death or the end of follow-up. Cases of death from other causes or those lost to follow-up were treated as censored cases. The study was approved by the Ethics Committee of Kitasato University School of Medicine (KMEO B15-21). Appropriate informed consent was obtained from all patients.

\section{Immunohistochemical Staining of S100A10}

After deparaffinizing in xylene, 3 - $\mu$ m-thick sections were rehydrated in a descending ethanol series and tap water, and then treated with $3 \%$ hydrogen peroxide for 10 min. Tissue sections were antigen-retrieved by autoclaving for $10 \mathrm{~min}$ in $0.01 \mathrm{M}$ citrate buffer ( $\mathrm{pH} 6.0$ ) with $0.1 \%$ Tween 20. After blocking with $0.5 \%$ casein for $10 \mathrm{~min}$, the sections were reacted with 1,000-times-diluted antiS100A10 polyclonal antibody (Abcam, Cambridge, UK) for $2 \mathrm{hr}$ at room temperature (RT). The sections were rinsed in Tris-buffered saline three times for 5 min each, and then allowed to react with ChemMate ENVISION (DAKO; Glostrup, Denmark) for $30 \mathrm{~min}$ at room temperature. The sections were subsequently visualized with Stable DAB solution (Invitrogen; Carlsbad, CA, USA) and counterstained with Mayer's hematoxylin. Negative controls were prepared by substituting phosphate-buffered saline for the anti-S100A10 antibody.

\section{Evaluation of Immunohistochemical Staining}

Only staining on the cytoplasmic membrane of the tumor cells was considered to be a positive result for S100A10. Vascular endothelial cells were used as an internal positive control (Figure 1A). The staining intensity was categorized into four groups by comparing the staining intensity of tumor cells with the positive control: $0=$ negative; 1 (weak)=weaker than the positive control; 2 (moderate) $=$ the same as the positive control; 3 (strong)=stronger than the positive control. Tumor with a staining score of 2 or 3 as well as positive staining in more than $5 \%$ of its total tumor cells were judged as positive. All of the immunostained sections were reviewed by two investigators (K.K. and S.Y.) without knowledge of the clinical data. Discordant cases were reviewed and discussed until a consensus was reached.

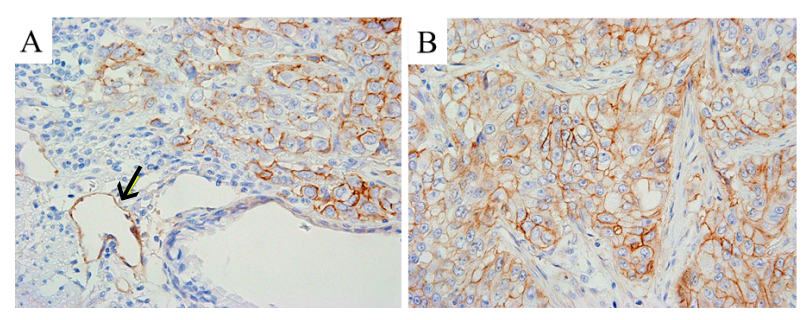

Figure 1. S100A10 Expression in Lung Adenocarcinomas. A) The normal vascular endothelial cells in the tumor stroma were used as an internal control for S100A10 expression (arrows). B) The vast majority of the tumor cells showed S100A10 expression at the membrane in lung adenocarcinoma. (original magnification: $\mathrm{A} \times 200 \mathrm{~B} \times 400$ )

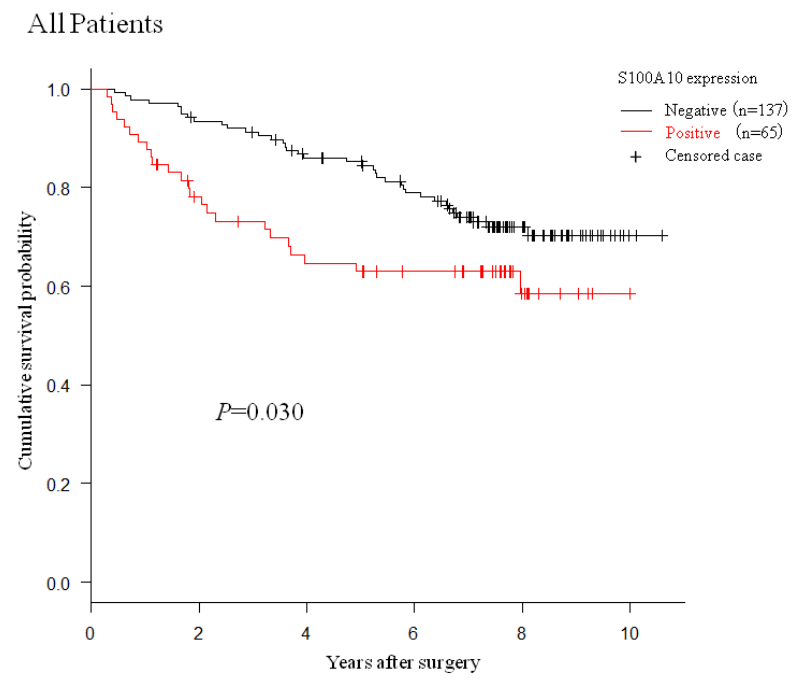

Figure 2. Cumulative Survival of Patients with Lung Adenocarcinoma According to S100A10 Expression Estimated by the Kaplan-Meier Method, Treating All Other Causes of Death and those Lost to Follow-up as Censored cases. S100A10 expression was significantly correlated with poorer survival in patients with lung adenocarcinoma 


\section{Statistical analysis}

Continuous variables are presented as the median (range), while numerical variables are given as $\mathrm{N}(\%)$. The relationships between S100A10 expression and clinicopathological parameters were assessed with Fisher's exact test. The cumulative survival of patients was estimated using the Kaplan-Meier method, and the significance of the survival differences between S100A10-positive and -negative groups was tested using the log-rank test. The 5-year cumulative survival probability was estimated using the life table method with the interval length set at 1 month. Multivariable analysis was performed by employing the Cox proportional hazards regression model to examine the interaction between S100A10 expression and other clinicopathological variables, and estimate the independent prognostic effect of S100A10 on survival by adjusting for confounding factors. The conventional P-value of 0.05 or less was used to determine the level of significance. All reported P-values are two-sided. All statistical analyses were performed with EZR (Saitama Medical Center, Jichi Medical University, Saitama, Japan), which is a graphical user interface for $\mathrm{R}$ (The R Foundation for Statistical Computing, Vienna, Austria). More precisely, it is a modified version of $\mathrm{R}$ commander designed to add statistical functions frequently used in biostatistics.

\section{Results}

\section{S100A10 Expression in Lung Adenocarcinoma}

Although S100A10 staining was observed simultaneously on the cytoplasmic membrane and in the cytoplasm of some tumor cells, the membraneous staining was much stronger and the majority of tumor cells showed only membraneous staining. (Figure 1B). Of the 202 surgically resected lung adenocarcinomas, 65 cases (32.2\%) were judged as positive for S100A10. S100A10 expression was also observed in vascular endothelial cells and fibroblasts in the tumor stroma. S100A10 was not detected in normal alveolar epithelial cells. No expression was observed in the negative controls.

\section{Clinicopathological Characteristics of Patients}

The clinicopathological characteristics of the 202 lung adenocarcinomas are summarized in Table 1 . The overall follow-up durations ranged from 4 to 129 months (median, 88 months). A total of 118 patients were alive at the end of the follow-up, 61 patients died of lung cancer, 8 patients died from other causes, and 15 patients were lost to follow-up. The causes of the 8 non-lung cancer deaths were pneumonia $(n=4)$, cholangiocellular carcinoma $(n=2)$, gastric cancer $(n=1)$, and leukemia $(n=1)$. None of these 8 patients died due to a surgery-related reason. Loss to follow-up of the 15 was due to discontinuing hospital attendance, and the durations of these patients' follow-up ranged from 15 to 87 months (median, 61 months).

\section{Relationship between S100A10 Expression and Clinicopathological Characteristics}

The relationships between S100A10 expression and clinicopathological characteristics are summarized in
Table 2. S100A10 expression was significantly correlated related with poorer tumor differentiation $(\mathrm{P}=0.015)$, higher stages of the disease (stages II and III) $(\mathrm{P}=0.004)$, and more frequent intratumoral vascular invasion $(\mathrm{P}=0.001)$. There was no significant correlation between S100A10 expression and the age, sex, smoking habit, tumor size, nodal status, or frequency of intratumoral lymphatic invasion or pleural invasion.

Kaplan-Meier Estimate of Survival in S100A10-Positive and-Negative Patients

All 202 patients with lung adenocarcinoma were included in the survival analysis. The overall follow-up periods ranged from 4 to 129 months (median, 87 months), and the 5 -year cumulative survival probability was $75 \%$ for all patients. Because a cumulative survival probability of $50 \%$ had not yet been reached, the overall median survival time was not determined. The 5 -year cumulative survival probability was $62 \%$ for the S100A10-positive group and $88 \%$ for the S100A10-negative group. While the median survival time was not available, the survival rate of the S100A10-positive group was significantly poorer $(\mathrm{P}=0.030)$ (Figure. 2).

Effect of S100A10 expression on patient's survival with uni- and multivariable analyses

Univariable analysis was performed according to the Cox proportional hazard model to evaluate the effect of S100A10 expression and other clinicopathological factors on survival. The p-TNM stage (HR, 6.99; 95\% CI, 3.98-12.27; $\mathrm{P}<0.001$ ), adjuvant chemotherapy (HR, 4.02; 95\% CI, 2.33-6.94; $\mathrm{P}<0.001)$, tumor differentiation

Table 1. Clinicopathological Characteristics of the Patients

\begin{tabular}{lc}
\hline Characteristics & Number of Patients $(\%)(\mathrm{N}=202)$ \\
\hline Age (years) & $63(37-82)$ \\
Median age (range) & $111(55.0)$ \\
$<65$ & $91(45.0)$ \\
$\geq 65$ & $108(53.4)$ \\
Sex & $94(46.6)$ \\
Male & \\
Female & $96(47.5)$ \\
Smoking habit & $106(52.5)$ \\
Never smoker & \\
Smoker & $113(55.9)$ \\
Tumor differentiation & $89(44.1)$ \\
Well & \\
Moderately/Poorly & $131(64.9)$ \\
p-TNM stage & $35(17.3)$ \\
StageI & $36(17.8)$ \\
StageII & \\
StageIII & $30(14.9)$ \\
Receiving adjuvant chemotherapy & $172(85.1)$ \\
Yes & \\
No & $118(58.4)$ \\
Vital status & $61(30.2)$ \\
Alive & $8(4.0)$ \\
Lung cancer-related death & $15(7.4)$ \\
Other cause of death & \\
Unknown & \\
\hline
\end{tabular}


Ken Katono et al

Table 2. Relationships between S100A10 Expression and Clinicopathological Parameters

\begin{tabular}{|c|c|c|c|c|}
\hline \multirow[t]{2}{*}{ Clinicopathological Parameters } & \multicolumn{2}{|c|}{ S100A10 Expression } & \multirow[t]{2}{*}{ Total } & \multirow[t]{2}{*}{ P-Value } \\
\hline & Positive $(\mathrm{N}=65)$ & Negative $(\mathrm{N}=137)$ & & \\
\hline Age, y; N (\%) & & & & 0.88 \\
\hline$<65$ & $35(31.5)$ & $76(68.5)$ & 111 & \\
\hline$\geq 65$ & $30(33.0)$ & $61(67.0)$ & 91 & \\
\hline Sex; N (\%) & & & & 0.132 \\
\hline Male & $40(37.0)$ & $68(63.0)$ & 108 & \\
\hline Female & $25(27.0)$ & $69(73.0)$ & 94 & \\
\hline Smoking habit; N (\%) & & & & 0.097 \\
\hline Never smoker & $25(26.0)$ & $71(74.0)$ & 96 & \\
\hline Smoker & $40(37.7)$ & $66(62.3)$ & 106 & \\
\hline Tumor differentiation; N (\%) & & & & 0.015 \\
\hline Well & $28(24.8)$ & $85(75.2)$ & 113 & \\
\hline Moderately/Poorly & $37(41.6)$ & $52(58.4)$ & 89 & \\
\hline p-TNM stage; N (\%) & & & & 0.004 \\
\hline Stage I & $33(25.2)$ & $98(74.8)$ & 131 & \\
\hline Stage II/III & $32(45.0)$ & $39(55.0)$ & 71 & \\
\hline Tumor size; N (\%) & & & & 0.113 \\
\hline$\leq 5 \mathrm{~cm}$ & $56(30.4)$ & $128(69.6)$ & 184 & \\
\hline$>5 \mathrm{~cm}$ & $9(50.0)$ & $9(50.0)$ & 18 & \\
\hline Nodal status; N (\%) & & & & 0.054 \\
\hline No & $43(28.3)$ & 109 (71.7) & 152 & \\
\hline $\mathrm{N} 1 / \mathrm{N} 2 / \mathrm{N} 3$ & $22(44.0)$ & $28(56.0)$ & 50 & \\
\hline Vascular invasion; $\mathrm{N}(\%)$ & & & & 0.001 \\
\hline Yes & $33(45.2)$ & $40(54.0)$ & 73 & \\
\hline No & $23(21.7)$ & $83(78.3)$ & 106 & \\
\hline Lymphatic invasion; $\mathrm{N}(\%)$ & & & & 0.05 \\
\hline Yes & $24(41.4)$ & $34(58.6)$ & 58 & \\
\hline No & $26(25.5)$ & $76(74.5)$ & 102 & \\
\hline Pleural invasion; N (\%) & & & & 0.337 \\
\hline Yes & $24(36.9)$ & $41(63.1)$ & 65 & \\
\hline No & $41(29.9)$ & $96(70.1)$ & 137 & \\
\hline
\end{tabular}

Table 3. Univariable and Multivariable Analyses of the Effect of S100A10 Expression on Survival

\begin{tabular}{|c|c|c|c|c|c|c|}
\hline \multirow[t]{2}{*}{ Factors } & \multicolumn{3}{|c|}{ univariate analysis } & \multicolumn{3}{|c|}{ multivariate analysis } \\
\hline & $\mathrm{HR}$ & $95 \% \mathrm{CI}$ & P-Value & HR & $95 \% \mathrm{CI}$ & P-Value \\
\hline \multicolumn{7}{|l|}{ S100A10 expression } \\
\hline Positive vs Negative & 1.75 & $1.05-2.93$ & 0.03 & 1.12 & $0.61-2.31$ & 0.60 \\
\hline \multicolumn{7}{|l|}{ Age } \\
\hline$\geq 65$ vs $<65$ & 1.26 & $0.76-2.09$ & 0.36 & $\mathrm{n} / \mathrm{d}$ & $\mathrm{n} / \mathrm{d}$ & $\mathrm{n} / \mathrm{d}$ \\
\hline \multicolumn{7}{|l|}{ Sex } \\
\hline Male vs Female & 0.83 & $0.50-1.38$ & 0.48 & $\mathrm{n} / \mathrm{d}$ & $\mathrm{n} / \mathrm{d}$ & $\mathrm{n} / \mathrm{d}$ \\
\hline \multicolumn{7}{|l|}{ Smoking habit } \\
\hline Smoker vs Never Smoker & 1.13 & $0.68-1.86$ & 0.64 & $\mathrm{n} / \mathrm{d}$ & $\mathrm{n} / \mathrm{d}$ & $\mathrm{n} / \mathrm{d}$ \\
\hline \multicolumn{7}{|l|}{ p-TMN stage } \\
\hline Stage II/III vs Stage I & 6.99 & $3.98-12.27$ & $<0.001$ & 3.42 & $1.66-7.05$ & $<0.001$ \\
\hline \multicolumn{7}{|l|}{ Adjuvant chemotherapy } \\
\hline No vs Yes & 4.02 & $2.33-6.94$ & $<0.001$ & 3.45 & $1.68-7.05$ & $<0.001$ \\
\hline \multicolumn{7}{|l|}{ Tumor differentiation } \\
\hline Moderately/Poorly vs Well & 3.08 & $1.82-5.23$ & $<0.001$ & 1.57 & $0.76-3.24$ & 0.22 \\
\hline \multicolumn{7}{|l|}{ Vascular invasion } \\
\hline Yes vs No & 5.48 & $3.00-9.99$ & $<0.001$ & 2.06 & $0.82-5.16$ & 0.12 \\
\hline \multicolumn{7}{|l|}{ Lymphatic invasion } \\
\hline Yes vs No & 4.43 & $2.48-7.92$ & $<0.001$ & 1.04 & $0.47-2.31$ & 0.91 \\
\hline \multicolumn{7}{|l|}{ Pleural invasion } \\
\hline Yes vs No & 3.35 & $2.02-5.56$ & $<0.001$ & 1.86 & $0.9-3.53$ & 0.06 \\
\hline
\end{tabular}

(HR, 3.08; 95\% CI, 1.82-5.23; $\mathrm{P}<0.001)$, vascular invasion (HR, 5.48; 95\% CI, 3.00-9.99; $\mathrm{P}<0.001$ ), lymphatic invasion (HR, 4.43; 95\% CI, 2.48-7.92; $\mathrm{P}<0.001)$, pleural invasion (HR, 3.35; 95\% CI, 2.02-5.56; $\mathrm{P}<0.001$ ), and S100A10 expression (HR, 1.75; 95\% CI, 1.05-2.93; $\mathrm{P}=0.03)$ were significant predictors of cancer-specific 
survival. However, when S100A10 expression and other clinicopathlogical variables including the p-TNM stage, adjuvant chemotherapy, tumor differentiation, vascular invasion, lymphatic invasion, and pleural invasion were included in multivariable analysis using the Cox proportional hazards regression model, S100A10 expression was not an independent predictor of a poorer survival (HR, 1.12; 95\%CI, 0.61-2.31; $\mathrm{P}=0.60)$. On the other hand, the p-TNM stage (HR, 3.42; 95\%CI, 1.667.05; $\mathrm{P}<0.001)$ and adjuvant chemotherapy (HR, 3.45; 95\% CI, 1.68-7.05; $\mathrm{P}<0.001)$ were independent predictors of a poorer survival (Table 3 ).

\section{Discussion}

In the present study, S100A10 was expressed in a subset of lung adenocarcinomas, and its expression was related to poorer tumor differentiation, a higher stage of disease, more frequent intratumoral vascular invasion, and a poorer prognosis, in keeping with the findings of Shang et al. in colorectal cancer (Shang et al., 2013).

S100A10 forms a heterotetramer with annexin A2 at the cell surface, which functions as a plasminogen receptor and facilitates the conversion of plasminogen to plasmin. Plasmin catalyzes the degradation of proteins of the basement membrane and ECM (Godier and Hunt, 2013). Therefore, S100A10 might contribute to the invasiveness of tumor cells by increasing plasmin production with consequent basement membrane and ECM degradation. Choi et al. reported that the HT1080 fibrosarcoma cell line, which has lost S100A10 expression, showed a marked decrease in invasiveness and the metastatic potential (Choi et al., 2003). Additionally, Zhang et al. (2004) reported similar findings in a CCL-222 colorectal cancer cell line, when S100A10 expression was downregulated (Zhang et al., 2004). These results argue for the contributions of S100A10 to cancer cell invasiveness. In the present study, S100A10 expression was significantly correlated with more frequent intratumoral vascular invasion, and there was also a clear tendency toward more frequent intratumoral lymphatic invasion, supporting the previously reported relationship between S100A10 and cancer cell invasiveness. S100A10 was also shown to contribute to cell spreading/migration, required for invasion of the local microenvironment. Sayeed et al. reported that the depletion of S100A10 using two different siRNAs induced the suppression of cell spreading in HeLa and MDA-MB 231 cell lines. Furthermore, they suggested that S100A10 could activate Rac1, which is essential for the promotion of cell spreading (Sayeed et al., 2013). Hence, the correlation of S100A10 expression and intratumoral lymphovascular invasion found in the present study might reflect the enhancement of cell spreading/ migration caused by S100A10.

S100A10 staining was detected in both the membrane and cytoplasm of some tumor cells, although the membraneous staining was much stronger and the majority of positive cells showed only staining on the cell membrane, suggesting the important function of S100A10 as a plasminogen receptor at the tumor cell surface.

There are also some reports of S100A10 being related to other processes of tumor metastasis and progression, including the migration of tumor-promoting macrophages into tumor sites, suppression of apoptosis in cancer cells, and regulation of angiogenesis (Hsu et al., 1997; Phipps et al., 2011; Surette et al., 2011). Further studies are needed to elucidate the multiple functions of S100A10 in various tumors.

In conclusion, we report for the first time that $\mathrm{S} 100 \mathrm{~A} 10$ is expressed in a subset of lung adenocarcinoma, and its expression is related to a poorer tumor differentiation, higher stage of the disease, more frequent intratumoral vascular invasion, and a poorer prognosis. Further studies are needed to elucidate the biological functions of S100A10 in lung adenocarcinoma in more detail, which might lead to the development of novel therapeutic strategies against lung adenocarcinoma.

\section{Acknowledgements}

This study was supported in part by JSPS KAKENHI Grant number 23590414.

\section{References}

Alberg AJ, Brock MV, Ford JG, et al (2013). Epidemiology of lung cancer: diagnosis and management of lung cancer, 3rd ed: american college of chest physicians evidence-based clinical practice guidelines. Chest, 143, 1-29.

Chen H, Xu C, Jin Q, et al (2014). S100 protein family in human cancer. Am J Cancer Res, 4, 89-115.

Choi KS, Fogg DK, Yoon CS, et al (2003). p11 regulates extracellular plasmin production and invasiveness of HT1080 fibrosarcoma cells. FASEB J, 17, 235-46.

Domoto T, Miyama Y, Suzuki H, et al (2007). Evaluation of S100A10, annexin II and B-FABP expression as markers for renal cell carcinoma. Cancer Sci, 98, 77-82.

Donato R (2001). S100: a multigenic family of calciummodulated proteins of the EF-hand type with intracellular and extracellular functional roles. Int J Biochem Cell Biol, 33, 637-68.

Godier A, Hunt BJ (2013). Plasminogen receptors and their role in the pathogenesis of inflammatory, autoimmune and malignant disease. J Thromb Haemost, 11, 26-34.

Goldstraw P, Crowley J, Chansky K, et al (2007). The IASLC lung cancer staging project: proposals for the revision of the TNM stage groupings in the forthcoming (seventh) edition of the TNM Classification of malignant tumours. J Thorac Oncol, 2, 706-14.

Hedhli N, Falcone DJ, Huang B, et al (2012). The annexin A2/ S100A10 system in health and disease: emerging paradigms. J Biomed Biotechnol, 2012, 406273.

Hsu SY, Kaipia A, Zhu L, et al (1997). Interference of BAD (Bcl-xL/Bcl-2-associated death promoter)-induced apoptosis in mammalian cells by 14-3-3 isoforms and P11. Mol Endocrinol, 11, 1858-67.

Ito Y, Arai K, Nozawa R, et al (2007). S100A10 expression in thyroid neoplasms originating from the follicular epithelium: contribution to the aggressive characteristic of anaplastic carcinoma. Anticancer Res, 27, 2679-83.

Kawai H, Minamiya Y, Takahashi N (2011). Prognostic impact of S100A9 overexpression in non-small cell lung cancer. Tumour Biol, 32, 641-6.

Kobayashi S, Boggon TJ, Dayaram T, et al (2005). EGFR mutation and resistance of non-small-cell lung cancer to gefitinib. $N$ Engl J Med, 352, 786-92. 


\section{Ken Katono et al}

Liu Y, Myrvang HK, Dekker LV, et al (2015). Annexin A2 complexes with $\mathrm{S} 100$ proteins: structure, function and pharmacological manipulation. Br J Pharmacol, 172, 1664-76.

Madureira PA, O'Connell PA, Surette AP, et al (2012). The biochemistry and regulation of S100A10: a multifunctional plasminogen receptor involved in oncogenesis. J Biomed Biotechnol, 2012, 353687.

Parkin DM (2001). Global cancer statistics in the year 2000. Lancet Oncol, 2, 533-43.

Phipps KD, Surette AP, O'Connell PA, et al (2011). Plasminogen receptor S100A10 is essential for the migration of tumorpromoting macrophages into tumor sites. Cancer Res, 71, 6676-83.

Sayeed S, Asano E, Ito S, et al (2013). S100A10 is required for the organization of actin stress fibers and promotion of cell spreading. Mol Cell Biochem, 374, 105-11.

Shang J, Zhang Z, Song W, et al (2013). S100A10 as a novel biomarker in colorectal cancer. Tumour Biol, 34, 3785-90.

Surette AP, Madureira PA, Phipps KD, et al (2011). Regulation of fibrinolysis by S100A10 in vivo. Blood, 118, 3172-81.

Tan Y, Ma SY, Wang FQ, et al (2011). Proteomic-based analysis for identification of potential serum biomarkers in gallbladder cancer. Oncol Rep, 26, 853-9.

Travis WD, Coby TV, Corrin B, et al (1999). World health organization international histological classification of tumors; histological typing of lung and pleural tumors. Springer.

Tsuna M, Kageyama S, Fukuoka J, et al (2009). Significance of S100A4 as a prognostic marker of lung squamous cell carcinoma. Anticancer Res, 29, 2547-54.

Wang H, Zhang Z, Li R, et al (2005). Overexpression of S100A2 protein as a prognostic marker for patients with stage I non small cell lung cancer. Int J Cancer, 116, 285-90.

Yoshino I, Yoshida S, Miyaoka E, et al (2012). Surgical outcome of stage IIIA- cN2/pN2 non-small-cell lung cancer patients in Japanese lung cancer registry study in 2004. J Thorac Oncol, 7, 850-5.

Zhang L, Fogg DK, Waisman DM (2004). RNA interferencemediated silencing of the S100A10 gene attenuates plasmin generation and invasiveness of Colo 222 colorectal cancer cells. J Biol Chem, 279, 2053-62. 